Revue européenne des migrations internationales

vol. $21-n^{\circ} 1 \mid 2005$

Femmes, genre, migration et mobilités

\title{
Genre, travail et migrations en Europe, Cahiers du CEDREF $n^{\circ} 10$
}

Catherine Quiminal

\section{(2) OpenEdition}

1 Journals

Édition électronique

URL : https://journals.openedition.org/remi/4251

DOI : 10.4000/remi.4251

ISSN : $1777-5418$

Éditeur

Université de Poitiers

Édition imprimée

Date de publication : 29 mai 2005

Pagination : 275-278

ISBN : 2-911627-39-3

ISSN : 0765-0752

Référence électronique

Catherine Quiminal, «Genre, travail et migrations en Europe, Cahiers du CEDREF n 10 », Revue européenne des migrations internationales [En ligne], vol. 21 - $n^{\circ} 1$ | 2005, mis en ligne le 10 septembre 2008, consulté le 14 avril 2022. URL : http://journals.openedition.org/remi/4251 ; DOI : https://doi.org/ 10.4000/remi.4251

Ce document a été généré automatiquement le 14 avril 2022.

(c) Université de Poitiers 


\title{
Genre, travail et migrations en Europe, Cahiers du CEDREF $n^{\circ} 10$
}

\author{
Catherine Quiminal
}

\section{RÉFÉRENCE}

Genre, travail et migrations en Europe, Cahiers du CEDREF n 10, décembre 2003, 159 p.

1 Les cahiers du CEDREF $^{1}$ persistent dans leur souci de faire connaître les travaux qui articulent rapports sociaux de genre et relations inter-ethniques. Faisant suite au numéro consacré aux femmes en migrations dont le thème central était les rapports sociaux de sexe et les stratégies différenciées des hommes et des femmes dans le processus migratoire, a paru au cours de l'hiver 2003/2004 un nouveau numéro: «Genre, travail et migrations en Europe ». L'ouvrage donne à voir, à travers les textes et des références bibliographiques abondantes, l'importance et l'intérêt des recherches qui abordent les mouvements migratoires, les politiques migratoires, la division internationale du travail à l'époque de la mondialisation, en termes de rapports de sexe.

2 Cette présentation de l'ouvrage n'entend pas redoubler l'excellente introduction qui en est faite par C. Zaidman avec la collaboration de Prisca Bachelet, soulignant l'intérêt d'un numéro européen, insistant sur la manière dont les femmes migrantes sont prises dans des rapports de dominations certes, mais également, circulent, travaillent, luttent pour faire respecter leurs droits, gagner en autonomie, pointant les nouvelles questions posées aux études féministes par la circulation du travail domestique et la professionnalisation de la reproduction.

3 Il s'agit, dans cette note de lecture, de mettre en évidence, sans aucune prétention à l'exhaustivité mais en fonction de mes compétences et de mes intérêts scientifiques, quelques éléments des débats théoriques qui traversent peu ou prou chacun des textes. Débats qui tant du côté des recherches féministes que du côté des recherches sur les 
migrations et les relations inter-ethniques, si tant est qu'elles soient séparables, sont loin d'être clos.

4 Trois grands ensembles de questions ont ainsi retenu mon attention.

1. Considérant le développement du secteur des services dans les pays occidentaux et l'accroissement des difficultés à survivre de nombreuses familles dans les pays du Sud qui conduisent de plus en plus de femmes à migrer, peut-on parler d'un nouveau système migratoire dans lequel les femmes migrantes occuperaient une place centrale?

2. Cette nouvelle configuration s'accompagne-t-elle non plus, comme c'était le cas lors de la période de la production de masse, d'une invisibilisation des femmes migrantes mais de leur minorisation, nouvelle forme de non-reconnaissance sociale, articulant ethnisme et sexisme?

3. Quelles conséquences théoriques et politiques découlent de l'occupation par des femmes migrantes d'une part importante des emplois dans le secteur des services, de leur prise en charge d'une partie des tâches de reproduction sociale dans un nombre grandissant de foyers?

\section{Migrations féminines et mondialisation}

Peut-on parler d'un nouveau système migratoire structuré autour de la mobilité des femmes s'accompagnant de représentations et de pratiques discriminatoires mais aussi de stratégies d'autonomie des femmes?

6 Si l'on met en regard les politiques migratoires sous leur double aspect : suspension de l'immigration économique, intégration des familles immigrées présentes sur les territoires nationaux d'un côté, et le manque de main-d'œuvre dans le secteur des services et dans certains secteurs des technologies de pointe de l'autre, on voit se dessiner un système complexe de rapports sociaux fondamentalement inégalitaires, inégalités justifiées par des arguments ethnistes et sexistes.

Dans de nombreux pays européens, la majorité des nouveaux arrivants légaux sont des femmes mettant en rapport des économies inégalement développées (E. Kofman, N. Ouali, A. Miranda, F. Scrinzi, L. Oso Casas) ${ }^{2}$. Ce qui n'a rien d'étonnant puisque l'obtention d'un droit au séjour se fait, à quelques exceptions près, au regard de critères familiaux et conjugaux (C. Lecellier). Pourtant, les femmes acceptées en tant que mères ou épouses n'ont guère d'autres issues que de rejoindre les rangs de l'immigration économique là où il y a de la demande, c'est-à-dire dans les services (S. Chaib).

De surcroît, la difficulté de survivre dans de nombreux pays du tiers monde (Asie, Afrique, Amérique Latine), la place qui leur est souvent assignée, voire les persécutions dont elles sont victimes (J. Freedman), poussent un nombre grandissant de femmes, informées des opportunités qu'offre une demande forte dans les domaines du travail domestique et plus généralement des services, à accepter des situations irrégulières assorties de rémunérations sans commune mesure avec le travail accompli et les minimums salariaux en vigueur dans les différents pays européens (F. Scrinzi, A. Miranda) contrevenant aux législations du travail, pouvant aller jusqu'à une forme «d'esclavage moderne » (N. Ouali). La «fermeture des frontières » aurait pour effet, sinon pour objectif, la mise à disposition dans les pays européens d'une main-d'œuvre mobile, bon marché, hors droits. De plus, à la différence des migrations des années 1960, certaines de ces femmes sont qualifiées et connaissent, au moins temporairement, 
une déqualification ce qui ne les empêche pas d'avoir des stratégies d'ascension sociale (E. Kofman).

9 Globalement on peut se demander si la transnationalisation du secteur des services ne relègue pas les femmes dans des secteurs toujours précaires et flexibles comme le montre dans plusieurs de ses travaux Saskia Sassen, accentuant les inégalités entre les hommes et les femmes à l'échelle mondiale, contribuant à l'ethnicisation des rapports sociaux (F. Scrinzi), à une hiérarchisation en raison non seulement du sexe mais des origines, une manière moderne de ne pas prendre en compte le coût du travail de reproduction domestique, laissant le soin aux femmes de régler le problème entre elles? De différer la question de l'égalité entre les hommes et les femmes en permettant aux femmes "modernes" de réaliser leur modernité grâce à l'aide de femmes tenues comme moins exigeantes parce que venues de contrées moins développées? Soulignons que tous les articles ont le souci de mettre en relation les effets de structure et les stratégies des femmes évitant par-là, misérabilisme à l'endroit des unes ou culpabilisation des autres.

10 Toutes les implications découlant de ce nouveau système de travail se déroulant à échelle mondiale ne sont pas encore étudiées, tant dans les pays de départ que dans les pays d'installation. Il convient notamment d'explorer tous les secteurs d'activité, tourisme, hôtellerie, commerce, médiation, animation socioculturelle, santé... dans lesquels les femmes migrantes sont actives (S. Chaib).

\section{De l'invisibilité à la minorisation des femmes migrantes}

11 Alors que dans de nombreux pays d'Europe le nombre d'entrée des femmes migrantes dépasse souvent celui des hommes, que signifie la place encore mineure, voire marginale, qui leur est faite tant par les politiques migratoires, qu'à travers les stéréotypes, les représentations dominantes? Que nous dit ce déni d'existence quant aux rapports sociaux de genre, de classe et de race?

12 Malgré la présence de plus en plus massive des femmes dans la migration, malgré leur apparition répétée sur la scène publique, leur regroupement en association, leur participation active à des luttes pour le logement, pour leurs droits, malgré le développement des recherches les concernant, les migrantes restent peu visibles. Toutefois la nature de leur invisibilité a changé. On peut se demander si, dans ce nouveau contexte, la notion d'invisibilité n'est pas défensive et s'il ne convient pas de parler de processus de minorisation soulignant ainsi les mécanismes de construction des différences et des rapports de domination. À cet égard une approche prenant en compte l'histoire d'un flux migratoire (N. Lillo) permet de comparer les différents enjeux sociétaux dont les femmes migrantes ont été dans le passé et sont aujourd'hui l'objet, d'intégrer dans les recherches la diversité des rapports de sexe et de domination variant selon les situations.

13 La représentation dominante de l'immigré est passée du travailleur jeune, force de travail non qualifié bon marché à celle du clandestin ou du jeune délinquant de banlieue. Cette figure plus volontiers masculine que féminine est renforcée par un certain nombre de stéréotypes concernant les femmes migrantes «mamans » venues dans le cadre du regroupement familial, femmes d'une autre époque, dépendantes de 
leurs époux, gardiennes des traditions, autant de stéréotypes qui confortent le point de vue selon lequel la présence de certains immigrés, notamment en France ceux des anciennes colonies, trop distants culturellement est peu souhaitable. Cette représentation des femmes migrantes a des répercussions sur l'ensemble des migrants. Elle contribue à racialiser l'immigration et la citoyenneté (C. Raissiguier). Alors que la mobilité des femmes est dans certains pays plus importante que celle des hommes (cf. Philippines, latino américaines) y compris des femmes seules, que les motifs de leur migration sont multiples, entre autres leur volonté d'autonomie ainsi que leurs stratégies d'ascension sociale, que leur travail n'est pas marginal mais fait système (S. Chaib), elles sont globalement présentées comme dominées donc mineures, dépendantes. Cette dépendance est renvoyée à leur culture, aux rapports de genre dans leur société d'origine, celle à laquelle elles sont censées « appartenir » et qui justifie les discriminations dont elles sont l'objet. Ce processus de minorisation renvoyant à des différences culturelles, dont les femmes seraient les victimes, apparaît comme particulièrement contradictoire lorsqu'on analyse le traitement réservé aux femmes demandant le statut de réfugiées (J Freedman).

\section{Plusieurs catégories de femmes}

14 Un point d'accord dans ce numéro et qui semble émerger dans les situations d'ethnicisation des groupes comme l'ont analysé les premières les féministes noires américaines : les femmes ne constituent pas un groupe homogène, il est nécessaire de prendre en compte la diversité des situations jusque et $\mathrm{y}$ compris celles où des femmes exploitent d'autres femmes, ont des intérêts divergents. Une telle affirmation ne contredit pas celle selon laquelle les femmes ont été socialement construites comme groupe social dominé, elle implique la déconstruction de la catégorie femme et l'introduction d'autres dimensions sociales, l'ethnicité, la race, la classe dans les analyses. Elle exige de s'interroger sur les orientations théoriques et militantes (lutte pour l'égalité) qu'ouvrent ces relations sociales de domination et d'exploitation entre femmes autochtones et femmes étrangères.

15 La prise en charge d'une partie du travail domestique par des femmes immigrées n'a-telle pas pour effet de pérenniser en le masquant un des fondements des inégalités entre les sexes? D'éluder la question des responsabilités familiales? L'investissement des femmes migrantes dans les tâches de reproduction sociale est d'autant moins reconnu qu'il repose sur des femmes dont la présence est illégale. Enfermées, voire cachées dans l'espace domestique ces femmes sont sans reconnaissance sociale, tenues hors espace public, hors politique. Elles se doivent de rester discrète, à discrétion d'autres femmes. Ces rapports de domination et d'exploitation, fortement ethnicisés, ne renforcent-ils pas l'idéologie de la différence si souvent invoquée pour justifier l'inégalité entre les sexes?

16 Enfin la dimension européenne de ce numéro des cahiers du CEDREF croisant la diversité des approches et des situations analysées, mettant en regard des articles traitant des rapports entre genre et politiques migratoires, mobilité des femmes et travail domestique, femmes migrantes et marché du travail nous invitent à explorer dans leur multiples dimensions, leur complexité, les rapports sociaux qu'engendrent les migrations féminines, les mécanismes de hiérarchisation qui en découlent. 
17 En effet, au cœur des problématiques surgit la question de l'articulation entre les rapports sociaux différemment instruits selon qu'ils relèvent d'une approche en termes de genre, de classe, d'origine ethnique ou de " race ", qu'ils privilégient une hiérarchie sociale ethniste, raciste, sexiste ou de classe. Une fois nommée, et le choix des mots n'est pas indifférent, articulation, intersectionnalité, imbrication, la question concernant la manière d'appréhender et de traiter des modalités, des effets de cette articulation, intersectionnalité, demeure.

18 Comment les différents rapports sociaux s'ordonnent-ils ? Y a-t-il un rapport qui prime sur les autres? Est-ce toujours le même ou diffère-t-il selon les situations, les niveaux d'analyse?

19 Ainsi les situations sont bien connues où la discrimination raciste freine ou interdit l'indépendance des femmes, leur affranchissement de l'assignation qui leur est faite au « communautarisme». Les discriminations dans l'accès à l'emploi, au logement, ne permettent guère d'assumer ou rendent plus problématique la rupture d'avec les siens. Le racisme des uns permet aux autres, les hommes et les femmes de la communauté par-là même renforcée, de rejeter celle qui va du côté de ceux qui la nient. Argument raciste fallacieux mais puissant des mères africaines qui incitent leur fille à prendre un époux du côté des dominés "Africains » plutôt que des dominants blancs racistes sur lesquels elles ne pourront agir en cas de conflits, de maltraitance par exemple.

20 L'ordre social raciste semble alors primer ou du moins entraver l'émergence de revendications féministes unifiées pour l'égalité des hommes et des femmes. La revendication d'égalité se transforme en revendication d'identité ce qui explique pour une part le petit nombre des chercheuses féministes françaises investies dans des recherches concernant les femmes en migration ${ }^{3}$.

21 Des modes de classement, de hiérarchisation, y compris entre les femmes, se croisent, se renforcent les uns les autres. D'où la nécessité en ce domaine plus qu'en tout autre d'éviter toute simplification des classifications et des cadres conceptuels, tout réductionnisme théorique (E. Koffman). De prendre en compte les différents registres, dont le sexe et la race, à travers lesquels les dominants font du consensus et du dissensus, de l'intégration et de la discrimination, de la hiérarchisation symbolique et pratique.

22 Comme tout rapport de pouvoir, les relations interethniques et le système de genre puisent les fondements de leur légitimation dans la nature, d'où la force et la récurrence des processus de naturalisation, l'importance pour l'ordre social dominant à ce que se croisent et se renforcent mutuellement les catégories de " race " et de sexe. Quand viennent à manquer les arguments de nature resurgissent les arguments d'altérité, les processus d'ethnicisation.

\section{NOTES}

1. CEDREF Case 7132, Université Paris 7 - Denis Diderot, 2 place Jussieu - 75251 Paris 
2. Les noms entre parenthèses renvoient aux articles des auteures dans le numéro des cahiers du CEDREF ici analysé.

3. Cf. entretien avec A. Golub, M. Morokvasic, C Quiminal par N. Barison et C. Catarino, Migrations sociétés, vol IX, n52, juillet-août 1997.

\section{AUTEUR}

CATHERINE QUIMINAL

URMIS-SOLIIS/Paris VII 